\title{
Mean Pulmonary Arterial Pressure Decreases with Exposure Time in the Tibet Plateau
}

\author{
Qian Chen" ${ }^{\#}$, Delong Duo", Yingjun Yan², Qirong Yan², Zhengxing $\mathrm{Ma}^{2}$, Qian $\mathrm{Li}^{2}$ and Yafeng \\ Wang $^{2 *}$ \\ ${ }^{1}$ Department of Medical College, Qinghai University, China
}

${ }^{2}$ Department of Pharmacy, Qinghai Provincial People's Hospital, China

*Corresponding author: Yafeng Wang, Department of Pharmacy, Qinghai Provincial People’s Hospital, China.

To Cite This Article: Qian Chen, Delong Duo, Yingjun Yan, Qirong Yan, Zhengxing Ma, Qian Li, Yafeng Wang. Mean Pulmonary Arterial Pressure Decreases with Exposure Time in the Tibet Plateau. Am J Biomed Sci \& Res. 2021 - 12(5). AJBSR.MS.ID.001793.

DOI: 10.34297/AJBSR.2021.12.001793.

Received: 眥 April 26, 2020; Published: 眥 April 30, 2021

\begin{abstract}
Hypoxic pulmonary hypertension (HPH) is a severe and progressive disease characterized by pulmonary vascular remodeling, increased pulmonary vascular resistance, right ventricular hypertrophy and, if not reversed, death. We maintained laboratory rats at two altitudes (2261 and 4300 meters, $75.2 \% \mathrm{O}_{2}$ and $58.5 \% \mathrm{O}_{2}$ ) on the Qinghai Tibetan Plateau and measured the changes in mean pulmonary pressure (mPAP) over two months. In the first month mPAP increased by $30 \mathrm{mmHg}$, while in the second month mPAP returned to normal. We hypothesized that the secondary decrease in mean pulmonary artery pressure was associated with the production of vasodilators, decreased cardiac output, and genes involved in adaptation to higher altitudes, including EGLN1, PPAR $\alpha$ and HIF- $2 \alpha$.
\end{abstract}

Keywords: High Altitude; Pulmonary Arterial Hypertension; Altitude Acclimatization

Abbreviations: HPH: Hypoxic Pulmonary Hypertension; mPAP: Mean Pulmonary Pressure; PVR: Pulmonary Vascular Resistance; RV: Ultimately Right Ventricular; HREs: Hypoxia Response Elements; SN: Single Nucleotide Polymorphism; RV: Right Ventricle; NO: Nitric Oxide; SNP: Single Nucleotide Polymorphism; SD: Standard Deviation; HAPC: High-Altitude Polycythemia; HPH: Hypoxic Pulmonary Hypertension; CMS: Chronic Mountain Sickness; eNOS: Nitric Oxide Synthase

\section{Introduction}

The Qinghai Tibet Plateau is one of the most extreme environments for human beings to live in, with an average altitude of over 4000 meters. There are about 140 million residents living on the plateau $[1,2]$. In addition, many visitors and tourists reach plateau altitudes due to tourism, physical training, national defense and trade contacts. In 2019, the number of tourists to Qinghai and Tibet will reach 50.8 million and 23.37 million people according to the Qinghai Provincial Cultural Tourism Bureau. Exposure to highaltitude can cause acute or chronic mountain sickness [3]. Hypoxic pulmonary hypertension (HPH) is a chronic mountain sickness with pulmonary vascular function and structure changes caused by hypoxia (with a prevalence of up to $18 \%$ ) [4,5] with a mean pulmonary artery pressure $>30 \mathrm{mmHg}$ at rest, which leads to the increased mean pulmonary arterial pressure (mPAP), pulmonary vascular resistance (PVR), and ultimately right ventricular (RV) heart failure and death [6,7]. We carried out experiments with laboratory rats in Maduo County, Qinghai Province, for two months, where the average altitude is more than 4300 meters. Right ventricular pressure and mPAP were measured by right cardiac catheterization. The results showed that the mPAP of these rats increased to $30 \mathrm{mmHg}$, providing a model for pulmonary arterial hypertension in rats. However, after a second month the mean pulmonary arterial pressure returned to normal, indicating that the rats had adapted to the high altitude. Recently specific genes and 
proteins have been identified as being important adapting to high altitudes for Tibetans and animals which have lived at high-altitude for prolonged periods. Elevated expression of EGLN1, PPAR $\alpha$ and HIF-2 $\alpha$ (EPAS1) in Tibetans and animals such as yaks and Tibetans sheep may help people and animals to survive in the high-altitude hypoxic environments [8-12].

The EGLN1, gene encodes for HIF-proline hydroxylase 2 (PHD2) and plays an important role in the HIF pathway as an oxygen sensor [13]. In recent years, studies have shown that EGLN1 demonstrate strong positive selection in people adapted to high-altitude hypoxia environments. HIF named hypoxia-inducible factors, it is a kind of nuclear protein that regulates transcription activity. HIF is an $\alpha / \beta$ elements (HREs) at target gene loci under hypoxic conditions and is involved in pulmonary vascular remodeling under hypoxia conditions and plays a central role in the occurrence of hypoxic pulmonary hypertension. It mediates transcription responses to hypoxia by regulating a number of genes involved in different pathways, including erythropoiesis, angiogenesis and glycolysis, thereby regulating the cellular oxygen content [14-18]. Although HIF-1 and HIF-2 can transcribe activate many of the same genes, they differ in their responses to hypoxia $[19,20]$. HIF-1 $\alpha$ represents the acute hypoxia response, while HIF- $2 \alpha$ /EPAS1 plays role in chronic hypoxia exposure through several genes involved in the cellular and systemic hypoxia response. EPAS1 encodes the hypoxia induction factor HIF-2, a transcription factor involved in the hypoxia response. EPAS1 has a single nucleotide polymorphism (SNP) at the beginning of the sixth exon. This SNP marks the location of splicing variant of alternative splicing of EPAS1 mRNA, which is expressed by humans at high altitudes. Humans living at high-altitude by activating a series of compensatory reactions occur in the respiratory system, sympathetic nervous system, cardiovascular system, central nervous system and blood system to alleviate the symptoms of hypoxia, a process known as highaltitude acclimatization [21,22]. The current scientific studies have shown that circulating metabolites of NO and NO derived in the lungs are significant factors for high-altitude adaptation [23]. In addition, nitric oxide (NO), an endothelium-derived vascular dilatation factor, can directly dilate blood vessels and maintain stable pulmonary circulation $[24,25]$. The vascular endothelium is the main site for the synthesis of NO, and endothelial nitric oxide synthase is the specific enzyme involved. Endogenous NO produced by endothelial nitric oxide synthase plays an important role in the relaxation of vascular tension [26]. In endothelial cells, nitric oxide (NO) as a major signaling molecules regulating pulmonary vasodilation, it is produced by endothelial nitric oxide synthase (eNOS) and triggers the soluble guanosine cyclase in vascular smooth muscle to produce cGMP, thus promote vasodilation [21,2730] eNOS is mainly distributed in endothelial cells and circulating blood cells. In the cardiovascular system, eNOS is a regulator of blood flow, leukocyte adhesion and migration, and an inhibitor of platelet activation and aggregation [30]. Some studies indicate that hypoxia exposure increases the activity and expression of eNOS [21].

As previously mentioned, these are all possible reasons for the decrease in mean pulmonary artery pressure. To determine if these factors played a role in the decrease in mPAP in rats after acclimation to high altitude, we looked at the expression of eNOS, EGLN1 and HIF- $2 \alpha$ and at the expression of BNP and ApoA5 to evaluate cardiac function. Patients with pulmonary arterial pressure, symptoms of right ventricular hypertrophy, right ventricular failure, and even death are caused by pulmonary arterial pressure overload, so RV failure is a major determinant of survival in HPH [31,32]. Active B-type natriuretic peptide (BNP) and the functionally inert N-terminal prohormone of BNP (NT-proBNP) are clinical biomarkers extensively used in $\mathrm{PAH}$ and heart failure and are used to evaluate cardiac function [33-35]. Apolipoprotein AV (ApoA5), a kind of the ApoA protein, is a key factor in regulating plasma triglyceride [36,37]. Research has shown that overexpression ApoA5 alleviates RV failure caused by pulmonary hypertension and inhibit the fibrosis of RV [31].

\section{Materials and Methods}

\section{Animals}

Twenty-four male Sprague-Dawley (SD) rats (specific pathogenfree; $140-180 \mathrm{~g}$ ) were obtained from the Animal Experimental Center of Xian Jiaotong University (Xian, China;) and were housed in a room with a $12 \mathrm{~h}$ dark-light cycle at $22 \pm 2^{\circ} \mathrm{C}$ and relative humidity at 45-65\%. Animal studies were approved by the Medical Ethics Committee of Qinghai Provincial People's Hospital (PHQP181102-01). Food and drinking water were provided ad libitum.

\section{Experimental Design}

Animals were randomly divided into 4 groups ( $n=6$ per group): two middle altitude groups (2261 meters) maintained for 30 days (Group A) or 60 days (Group B); and two high-altitude groups (4300 meters) maintained for 30 days (Group C) or 60 days (Group D).

\section{Hemodynamic Measurements}

At the end of the experimental periods the rats were anaesthetized with pentobarbaital sodium (i.p., 30mg/kg) and body weight was recorded. The mean pulmonary arterial pressure (mPAP) was determined by right cardiac catheterization. The right ventricle (RV), left ventricle (LV) and interventricular septum (S) of each rat was separated at the ventricular septal edge. The weight was measured for each rat and was used to determine the right ventricle index (RV/LV+S, RVHI) [38-40]. 


\section{Tissue Harvest and Histomorphology}

Assay At the end of experiment, the lung, liver, kidney and heart tissues were collected and fixed in 4\% paraformaldehyde for 2 days, followed by dehydration with gradient alcohol, paraffin embedding and sectioning. Morphological changes were observed with an optical microscope (RX50, Ningbo Shun-yu Instrument Co. LTD, Ningbo, China) using hematoxylin \& eosin (H\&E) staining.

\section{Real Time Quantitative PCR Analysis}

Total mRNA from the lung, liver, kidney and heart were isolated using a total RNA extraction reagent (YESEN, Shanghai, China) following the manufacturer's protocol. The TRIeasyTM Total RNA Extraction Reagent (YESEN, Shanghai, China) and the Hifair $1^{\text {st }}$ Strand cDNA Synthesis SuperMix (YESEN, Shanghai, China) with qPCR. SYBR Green Master Mix (YESEN, Shanghai, China) were used on an ABI-7900HT system (Applied Biosystems, USA) for real-time quantitative PCR (RTqPCR). mRNA levels were calculated using the comparative $\mathrm{Ct}$ method $(\Delta \Delta \mathrm{Ct})$ after normalization to GAPDH. The primers used for RTqPCR in this study were as the follows: HIF- $2 \alpha$ (forward

$$
\begin{aligned}
& \text { primer:5'-TCACTCATCCTTGCGACCAC-3', reverse } \\
& \text { primer:5'-CAGGTGGCCGACTTAAGGTT-3'); EGLN1(forward } \\
& \text { primer:5'-AGGGCTAACGCTAATCACCT-3', reverse } \\
& \text { primer:5'-TTGTTGTCTTGAGACGCAGC-3'); eNOS (forward } \\
& \text { primer:5'-CCCAGGAGAGATCCACCTCA-3', reverse } \\
& \text { primer:5'-CGGAAGGGTGCAATACCAGT-3'); ApoA5(forward } \\
& \text { primer:5'-CACTCCCGTGGCTTCTAGTG-3', reverse } \\
& \text { primer:5'-GGACTGGCGAGCCTTAGTTT-3'); VEGF (forward } \\
& \text { primer:5'-GCAGCGACAAGGCAGACTAT-3', reverse } \\
& \text { primer:5'-GAGGGAGTGAAGGAGCAACC-3'); BNP (forward } \\
& \text { primer:5'-AGTCTCCAGAACAATCCACGATGC-3', reverse }
\end{aligned}
$$

primer:5'-CCGGAAGGCGCTGTCTTGAG-3'); TGF- $\beta R$ (forward

$$
\text { primer:5'- GCGATCTAACCTGTTGCCTGTG-3', reverse }
$$

primer: 5'-GGGCCATGTATCTCGCTGTTC-3'); GAPDH (forward

primer:5'-AATGGTGAAGGTCGGTGTGAAC-3', reverse

primer:5'-AGGTCAATGAAGGGGTCGTTG-3').

\section{Statistics Methods}

All results were performed by using SPSS (version 20.0) software and presented as mean \pm standard deviation (SD). One-way analysis of variance (ANOVA) was applied to determine statistical significance. Pair comparison between groups was analyzed by LSD-t test, and Dunnett's method was used to analyze when the variance was uneven. The data were considered statistically significant when $\mathrm{p}$-value $<0.05$.

\section{Results}

Changes in Pulmonary Hemodynamics and Ameliorated Right Ventricle Remodeling in PAH Rats The mPAP and RVHI in the group $C$ increased significantly as compared to the group $\mathrm{A},(\mathrm{P}<0.05$, (Figure 1A \&1B) indicating that a PAH models was established. The mPAP and RVHI in the group D decreased markedly when compared to group $C(P<0.05)$ (Figure $1 \mathrm{~A} \& 1 \mathrm{~B})$. Comparing group A to group $\mathrm{B}$, the mPAP and RVHI were no significant difference. The mPAP and RVHI were in normal range at the middle altitude. The Pathological Changes in Hypobaric Hypoxia-induced PAH Rats The middle-altitude 30-day group showed normal structure and morphology of the lung, liver, kidney and heart as determined by H\&E staining (Figure 2A-2D). However, in the second month, the middle altitude group pulmonary artery medial thickness and RVHI values increased (Figure 2A), but the liver, kidney and heart tissue showed normal structure and morphology (Figure 2B-2D). Compared to the middle altitude group in the first month, the highaltitude group 1 showed increased pulmonary artery thickening with proliferation of intermediate smooth muscle cells.
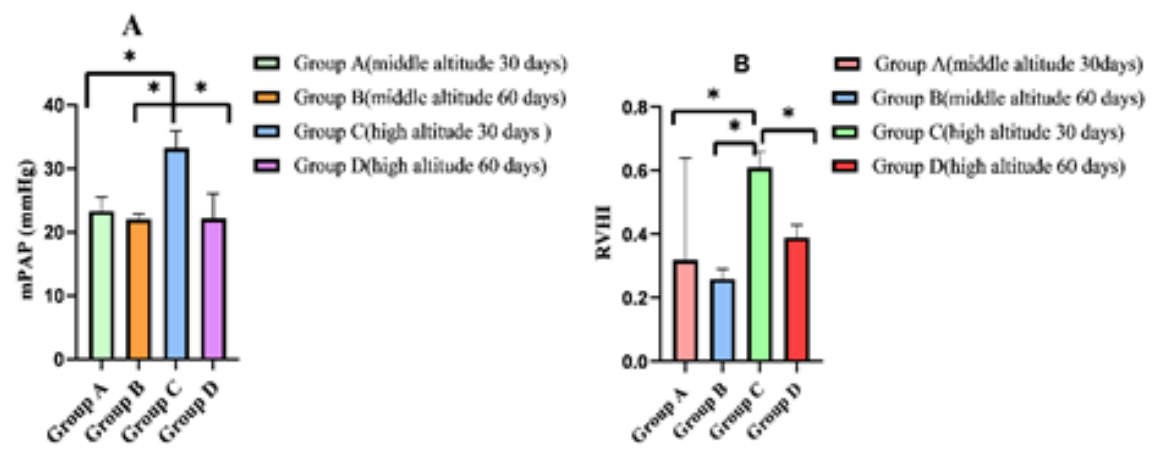

Figure 1: mPAP $(\mathrm{A})$ and $\mathrm{RVHI}(\mathrm{B})$ of rats in the middle and high elevation groups. ${ }^{*} \mathrm{P}<0.05$ 


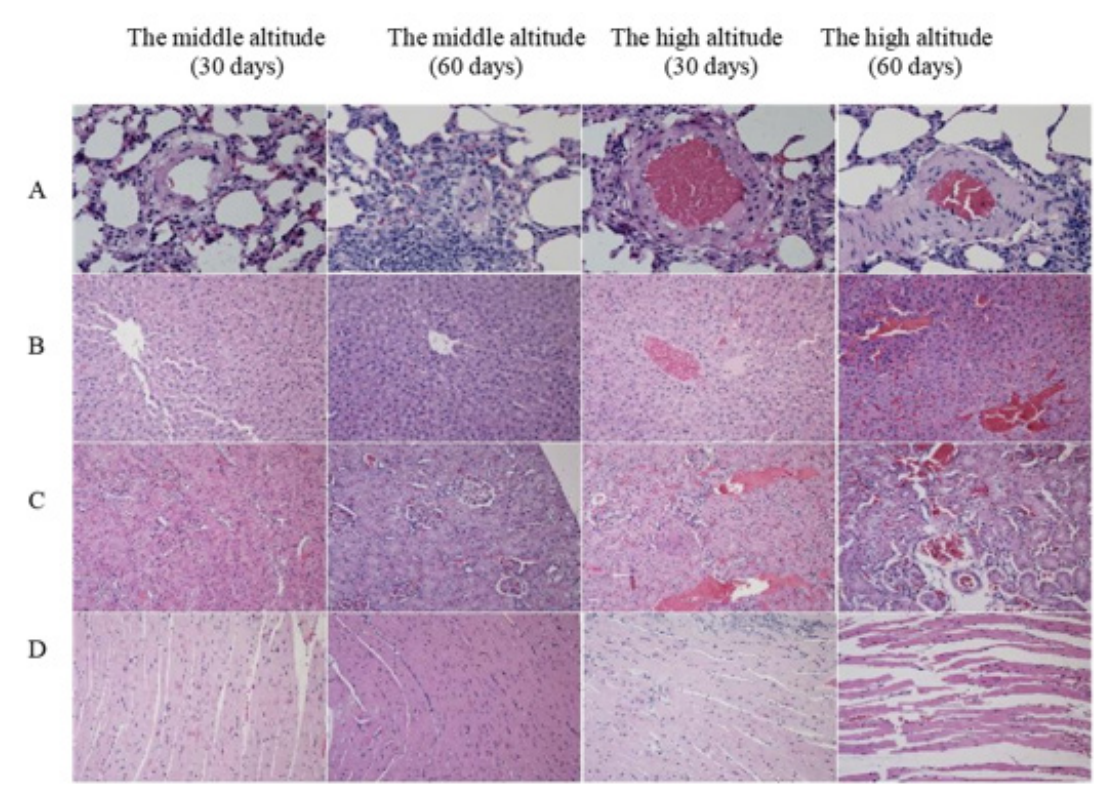

Figure 2: H\&E staining of the main organs in the different groups (400x). Representative images are shown in (A) Lung, (B) liver, (C) kidney and (D) heart.

The alveolar septum was broken and hemorrhaging was evident (Figure 2A), the arrangement of hepatocytes was disordered, some sinusoidal hemorrhage was evident, and the central venous congestion was severe (Figure 2B). In the kidney the glomerular basement membrane was thickened, and renal tubular epithelial cells were oedema with vacuolar degeneration (Figure 2C), the renal tubular lumen was dilated. The myocardial fiber boundary was not clear, the myocardial fiber arrangement was disordered and hemorrhage was readily evident (Figure 2C). Upon comparison of the two high-altitude group (1 and 2) showed that with the extension of time, the wall of pulmonary arterioles gradually thickened, and the proliferation of intermediate smooth muscle cells was obvious, hemorrhage was considerable with inflammatory cell infiltration (Figure 2A). Congestion of hepatic sinuses and central veins was also considerable and hemorrhage was more serious at 60 days than at 30 days (Figure 2B).

The kidney glomerular basement membrane gradually thickened, renal tubular epithelial cells became edematous, renal tubular epithelial cells demonstrated visible cytoplasmic vacuolar degeneration, with considerable hemorrhage at 60 days, more so than at 30 days (Figure $2 \mathrm{C}$ ), there were obvious enlargement of myocardial space, disordered arrangement, infiltration of inflammatory cells and rupture of myocardial fibers (Figure 2D). Real-time PCR analysis Real-time PCR was performed to quantify mRNA levels of BNP, ApoA5, eNOS, HIF-2 $\alpha$, EGLN1, VEGF and TGF$1 \beta$. Glyceraldehyde-3-phosphate dehydrogenase (GAPDH) served as a loading control. Under altitude hypoxia, altitude adaptation can include the expression of eNOS in lung and heart tissue. As shown in Figure 3A, in the lung tissue the expression of eNOS in the middle altitude groups was significantly higher than that of the highaltitude group during the first month and second months $(\mathrm{p}<0.05)$. In the high-altitude groups and the middle altitude groups the expression of eNOS in the second month was higher than the first month. In the heart tissue, (Figure 3B) the expression of eNOS in the high-altitude groups was significantly lower than the middle altitude groups during the first month and second month $(\mathrm{p}<0.05)$. In the high-altitude and middle altitude groups, in comparing the expression of eNOS in first month and second month, the expression of eNOS in the second months was higher than in the first month $(\mathrm{p}<0.05)$. Rats exposed to high-altitude hypoxia for a prolonged period may have elevated expression of genes that reflect altitude adaptation. The expression of EGLNI and HIF- $2 \alpha$ can reflect altitude adaptability. In the lung tissue, the expression of EGLN1 (Figure 3C) and HIF-2 $\alpha$ (Fig.3D) in the high-altitude groups was significantly higher during the second month than in the first month. Expression of EGLN1 in the high-altitude groups was significantly higher than the middle altitude group ( $\mathrm{p}<0.05)$. On the contrary, the expression of HIF-2 $\alpha$ (Figure 3D) in the high-altitude groups was lower than that of the middle altitude groups.

In the kidney tissues (Figure 3E), the expression of EGLN1 in the high-altitude group and the middle altitude group, during the second month was higher than the first month $(\mathrm{p}<0.05)$. In the liver tissues (Figure 3F), the expression of EGLN1 in the high-altitude group and the middle altitude group, during the second month was lower than in the first month $(\mathrm{p}<0.05)$. These results demonstrated that when rats are exposed to low oxygen and high-altitude for a 
prolonged period elevated expression of EGLN1 may help the rats better adapt to altitude hypoxia, which may be one of the reasons for the decrease of average pulmonary artery pressure in rats during the second month. In the lung tissue (Figure 3D), the expression of HIF- $2 \alpha$ in the high-altitude group 2 and middle altitude group 4 during the second month was higher than in the first month group
(1 and 3). During the first month and second month, the expression of HIF- $2 \alpha$ in the high-altitude groups was lower than the middle altitude groups. There was no obvious difference in the expression of HIF- $2 \alpha$ between the high-altitude group and the middle altitude groups in the first and second month.

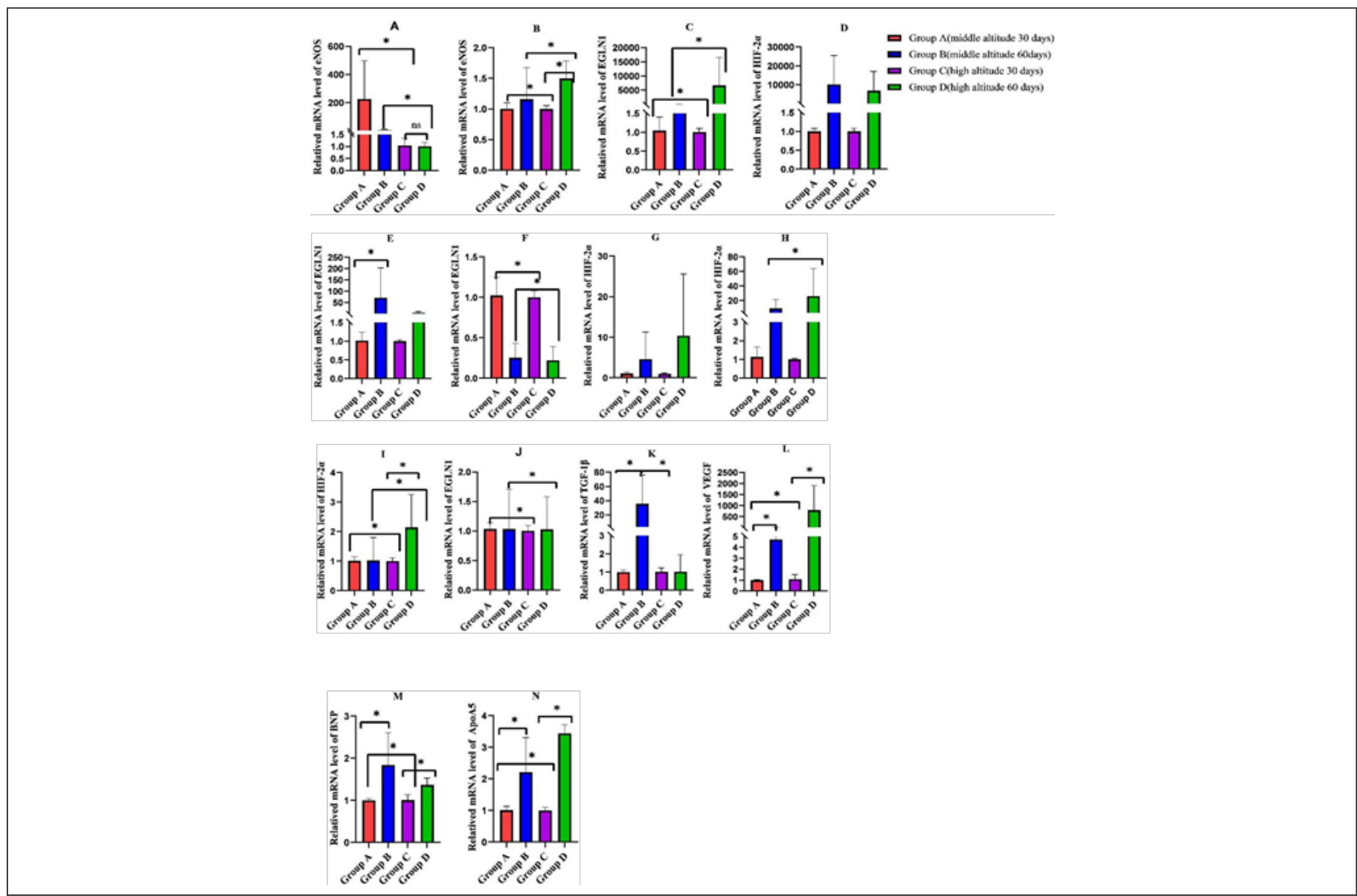

Figure 3: Bar graph showing the results of relatived mRNA levels of eNOS in the lung (A) and heart(B) tissues. Relatived mRNA levels of EGLN1 in the lung $(C)$, kidney $(E)$, liver $(F)$ and heart $(J)$ tissues. Relatived mRNA levels of HIF-2 $\alpha$ in the lung $(D)$, liver $(G), \operatorname{kidney}(H)$ and heart $(I)$ tissues. Relatived mRNA levels of TGF-1 $\beta(K)$ and VEGF $(L)$ in the lung tissue. Relatived mRNA levels of $B N P(M)$ and $A p o A 5(N)$ in the heart tissue. ${ }^{*} P<0.05$.

In the liver (Figure 3G) and kidney tissues (Figure 3H), the expression of HIF-2 $\alpha$ in the high-altitude groups in the second month was significantly higher than in the first month. In the heart (Figure 3I), and kidney tissues (Figure 3H), in the first and second month, the expression of HIF- $2 \alpha$ in the middle altitude groups was higher than the high-altitude group $(p<0.05)$. In the liver tissue (Figure 3G), the expression of HIF-2 $\alpha$ in the middle altitude group 3 was higher than the high-altitude group 1 during the first month. On the contrary, in the second month, it was lower than the high-altitude group. In the heart tissue (Figure 3J), during the first and second month, the expression of EGLN1 in the middle altitude groups was higher than the high-altitude groups $(\mathrm{p}<0.05)$, there was no obvious difference expression of EGLN1 between the first and second months (Figure 3J). These results demonstrated that HIF- $2 \alpha$ appears to play an important role in the adaptation to hypoxia at high altitude. We found high expression of EGLN1, HIF$2 \alpha$ and eNOS in the lung and heart tissues, these findings which provided evidence that EGLN1, HIF- $2 \alpha$ and eNOS may be the cause of decreasing mean pulmonary arterial pressure. In lung tissue during the first month the expression of TGF-1 $\beta$ (Figure 3K) in the high-altitude group was significantly higher than the middle altitude group $(\mathrm{p}<0.05)$, and in the second month it was lower than the middle altitude group. There was no significant difference in expression of TGF-1 $\beta$ between the first and second month in the high-altitude groups. In the middle altitude groups the expression of TGF-1 $\beta$ during the second month was markedly higher than in the first month $(\mathrm{p}<0.05)$. The expression of VEGF (Figure $3 \mathrm{~L})$ in the high-altitude group and middle altitude group was higher in 
the second month than the first month $(\mathrm{p}<0.05)$. During the first month, the expression of VEGF in the high-altitude group was lower than the middle altitude group $(\mathrm{p}<0.05)$. In contrast, in the second month expression in the high-altitude group was higher than in the middle altitude group. These results showed that the high expression of VEGF and TGF- $1 \beta$ in the lung tissue, demonstrate that after long-term exposure to hypoxia at high-altitude is associated with thickening of the vascular wall of lung tissue, vascular smooth muscle hyperplasia and pulmonary vascular remodeling. In heart tissue during the first month and second month the expression of BNP (Figure 3M) in the high-altitude groups was lower than in the middle altitude groups $(\mathrm{p}<0.05)$. The expression of BNP in the highaltitude groups and middle altitude groups was significantly higher in the second month than in the first month $(\mathrm{p}<0.05)$. During the first month and second month, the expression of ApoA5 (Figure 3N) in the high-altitude groups was lower than in the middle altitude group $(\mathrm{p}<0.05)$. The expression of ApoA5 in the high-altitude and middle altitude groups in the second month was significantly higher than in the first month $(\mathrm{p}<0.05)$.

\section{Discussion}

Chronic mountain sickness (CMS) is a symptom of maladjustment to living at high-altitude and often occurs in people who live in areas over $3000 \mathrm{~m}$ above sea level for a long time. CMS is characterized by high-altitude polycythemia (HAPC) and hypoxic pulmonary hypertension (HPH). In this study, we did Tibetan Plateau field experiments (altitude about $4300 \mathrm{~m}$ ) to study HPH by establishing a rat model of pulmonary hypertension. The rats were maintained fed for 30 days and 60 days, after which we measured mPAP and RVHI, mPAP increased in the first month but returned to normal by the end of the second month. Therefore, we explored the possible causes of this phenomenon. In our experiment, the HIF- $2 \alpha$, EGLN1, eNOS, VEGF, TGF-1 $\beta$, BNP, ApoA5 mRNA were detected by qPCR. The results showed that in the lung and heart tissues, the expression of eNOS in the second month was higher than that in the first month.

This result demonstrates that prolonged exposure to highaltitude will induce endothelial nitric oxide synthase (eNOS) and increase NO, dilating pulmonary vessels and reducing pulmonary vessel pressure. Nitric oxide (NO) as a major signaling molecules regulating pulmonary vasodilation, it is catalyzed by endothelial nitric oxide synthase (eNOS). NO activates guanosine cyclase which catalyzes the concersion of guanosine triphosphate (GTP) into cyclic guanosine phosphate (cGMP). cGMP activates cGMP-dependent protein kinase (PKG), namely sGC /cGMP /PKG pathway, to reduce intracellular calcium ion concentration and thereby vasodilate pulmonary vascular smooth muscle $[41,42]$. We speculate that the high expression of eNOS in rat lung and heart tissue after prolonged altitude hypoxia contributes to the long-term survival in the plateau area, and may be the reason for the decrease of mean pulmonary artery pressure. The Qinghai Tibet Plateau is an inland plateau in Asia, the largest plateau in China and the highest in the world. Research has found that people who live on the plateau can adapt to the high-altitude environment, resulting in better aerobic exercise ability, more effective oxygen transport efficiency, greater resting ventilation, higher oxygen saturation, lower hemoglobin level and lower pulmonary vasoconstrictive reactivity in an hypoxia environment [43]. In recent years, genes have been identified in Tibetans and yaks, which may help them better survive at high altitude, including EPAS1/HIF-2 $\alpha$, EGLN1/PHD2, PPAR $\alpha$ etc $[8,44,45]$. In our study we found that the expression of EGLN1 and HIF- $2 \alpha$ in rats maintained at high-altitude was higher in the second month than in the first month, suggesting a continuing adaption to altitude hypoxia, and suggest that EGLN1 and EPAS1/HIF- $2 \alpha$ are significant genes involved in this hypoxia response. The latter is related to positive regulation induced by hypoxia and is the key regulatory factor of chronic hypoxia-related genes, and is involved in the upstream regulation of EPO, VEGF and eNOS-related genes [46].

EPAS1 is abundant in lung and placenta, which are the key tissues for oxygen exchange between adult and fetus [47]. Some studies have indicated that EPAS1 is related to the hypoxia induction pathway, and the level of its expression can be significantly increased in an hypoxia environment. NO is an effective vasodilator and the key to its production is the enzyme eNOS, the transcription of which has been shown to be induced by the interaction between EPAS1-expressed HIF2 and two adjacent HRE sites on the heNOS promoter [48]. EGLN1 and HIF- Proline hydroxylase 2 (PHD2) expression was also up-regulated by hypoxia. Several recent studies have shown that, due to the strong positive selection in the high-altitude and low-oxygen environment adapted populations, EGLNI, a regulatory gene of HIF, plays a key role in the regulation of various subtypes of HIF, and becomes an important gene related to altitude adaptation and hemoglobin concentration in various plateau adaptation populations, including Tibetans. EGLN1 and EPAS1/HIF-2 $\alpha$ may contribute to the decrease in mean pulmonary artery pressure.

When we compared the high-altitude group with the middlealtitude group, we found that the expression of eNOS, EGLN1, HIF- $2 \alpha$ in the middle-altitude groups was higher than the highaltitude groups. This finding is inconsistent with many other relevant studies. We speculate that this may be related to the different altitudes of rats, and perhaps the adaptability of rats at different altitudes is also different. Further research is needed on this issue. Cardiac function was evaluated by testing BNP, and the results showed that the expression of BNP in the second month was higher than in the first month. Combined with the results of HE staining, it means that the heart injury in the second month is more serious in both the high and middle-altitude groups. A high level of BNP indicates poor cardiac function, as a result, the ability of the ventricles to contract decreases, reducing cardiac output into the 
pulmonary artery, thereby reducing pulmonary vascular resistance. So even though mean pulmonary artery pressure decreased in the rats, the damaged to the heart eventually led to death. We measured ApoA5 levels and the results showed that the expression of ApoA5 in the second month was higher than the first month. The overexpression of ApoA5 has been shown to alleviates PAH and RV failure and inhibit the fibrosis of the RV. [31]. However, our results showed that high expression of BNP was also accompanied by high expression of ApoA5, indicating that protective factors and damage factors are both expressed. Combined with H\&E staining, we speculated that the expression of injury factors might be higher than that of protective factors, which needs to be further studied. TGF- $\beta 1$ is a multifunctional cytokine that regulates cell growth, differentiation and proliferation in vivo [49] TGF- $\beta$ can inhibit the expression of phosphatase and tensinophilic deleted on chromosome 10 (PTEN) and promote pulmonary vascular remodeling and thickening [50]. The expression of TGF- $\beta$ in rats exposed to hypoxia increased significantly. VEGF plays a protective role in early PAH. The increase in VEGF in hypoxia tissue promotes angiogenesis, which is beneficial for the body to adapt to a hypoxia environment and ensures that oxygen and nutrients are delivered to hypoxia cells [51-53]. Under a hypoxia environment, the hypoxia response element in the VEGF gene promoter directly participates in pulmonary vascular remodeling by stimulating HIF- $1 \alpha$ and a complex signal transduction pathway, which leads to an increase in pulmonary arterial pressure. Our results show that the expression of VEGF and TGF- $\beta$ were both increased. This indicates that although the mean pulmonary artery pressure of the rats decreased at the second month, the proliferation of pulmonary vascular endothelial cells induced vascular proliferation.

\section{Conclusion}

In this study, we explored the possible causes of the decrease in mean pulmonary artery pressure after prolonged hypoxia in rats, but we were not able to determine which factor or pathway was responsible for the decrease. Our experiment lasted for two months, and we found that the average pulmonary artery pressure decreased at two months after being elevated at one month. Longer term studies are needed to understand this phenomenon.

\section{Acknowledgments}

This research was funded by Science and Technology Plan Project of Qinghai Province (2020-SF-131).

\section{Conflict of Interest}

The authors declare no conflict of interest.

\section{References}

1. Boucly A, Weatherald J, Savale L, Jaïs X, Cottin V, et al. (2017) Risk assessment, prognosis and guideline implementation in pulmonary arterial hypertension. The European respiratory journal 50(2): 1700889.
2. West JB (2012) IX World Congress on High Altitude Medicine and Physiology, Taipei, Taiwan, November 3-6, 2012. High altitude medicine \& biology 13(3):140.

3. Barry PW, Pollard AJ (2003) Altitude illness. BMJ Clinical research 326: 915-919.

4. Mirrakhimov AE, Strohl KP (2016) High-altitude Pulmonary Hypertension: an Update on Disease Pathogenesis and Management. The open cardiovascular medicine journal 10: 19-27.

5. Gao X, Zhang Z, Li X, Li C, Hao J, et al. (2020) Macitentan Attenuates Chronic Mountain Sickness in Rats by Regulating Arginine and Purine Metabolism. J Proteome Res 19(8): 3302-3314.

6. Lewis RA, Durrington C, Condliffe R, Kiely DG (2020) BNP/NT-proBNP in pulmonary arterial hypertension: time for point-of-care testing? European respiratory review: an official journal of the European Respiratory Society 15(156): 200009.

7. Chen J, Sysol JR, Singla S, Zhao S, Yamamura A, et al. (2017) Nicotinamide Phosphoribosyltransferase Promotes Pulmonary Vascular Remodeling and Is a Therapeutic Target in Pulmonary Arterial Hypertension. Circulation 135(16): 1532-1546.

8. Simonson TS, Yang Y, Huff CD, Yun H, Qin G, et al. (2010) Genetic evidence for high-altitude adaptation in Tibet. Science 329: 72-75.

9. Buroker NE, Ning XH, Zhou ZN, Li K, Cen WJ, et al. (2012) EPAS1 and EGLN1 associations with high altitude sickness in Han and Tibetan Chinese at the Qinghai-Tibetan Plateau. Blood cells, molecules \& diseases 49(2): 67-73.

10. Lorenzo FR, Huff C, Myllymäki M, Olenchock B, Swierczek S et al. (2014) A genetic mechanism for Tibetan high-altitude adaptation. Nature genetics 46(9): 951-956.

11. Xiang K, Ouzhuluobu, Peng Y, Yang Z, Zhang X, et al. (2013) Identification of a Tibetan-specific mutation in the hypoxic gene EGLN1 and its contribution to high-altitude adaptation. Molecular biology and evolution 30(8): 1889-1898.

12. Yi X, Liang Y, Huerta-Sanchez E, Jin X, Cuo ZX (2010) Sequencing of 50 human exomes reveals adaptation to high altitude. Science 329: 75-78.

13. Mishra A, Mohammad G, Thinlas T, Pasha MA (2013) EGLN1 variants influence expression and $\mathrm{SaO}_{2}$ levels to associate with high-altitude pulmonary oedema and adaptation. Clinical science 124(7): 479-489.

14. Smith TG, Robbins PA, Ratcliffe PJ (2008) The human side of hypoxiainducible factor. British journal of hematology 141(3): 325-34.

15. Semenza GL (2009) Regulation of oxygen homeostasis by hypoxiainducible factor 1. Physiology 24: 97-106.

16. Van Patot MC, Gassmann M (2011) Hypoxia: adapting to high altitude by mutating EPAS-1, the gene encoding HIF- $2 \alpha$. High altitude medicine \& biology 12(2): 157-167.

17. Berra E, Benizri E, Ginouvès A, Volmat V, Roux D, et al. (2003) HIF prolylhydroxylase 2 is the key oxygen sensor setting low steady-state levels of HIF-1alpha in normoxia. The EMBO journal 22(16): 4082-4090.

18. Fandrey J, Gorr TA, Gassmann M (2006) Regulating cellular oxygen sensing by hydroxylation. Cardiovascular research 71(4): 642-651.

19. Camenisch G, Stroka DM, Gassmann M, Wenger RH (2001) Attenuation of HIF-1 DNA-binding activity limits hypoxia-inducible endothelin-1 expression. Pflugers Archiv: European journal of physiology 443(2): 240-249.

20. Loboda A, Jozkowicz A, Dulak J (2010) HIF-1 and HIF-2 transcription factors similar but not identical. Molecules and cells 29(5): 435-442.

21. Pooja, Sharma M, Singh K, Himashree G, Bhaumik G, et al. (2020) Estrogen receptor (ESR1 and ESR2)-mediated activation of eNOS-NOcGMP pathway facilitates high altitude acclimatization. Nitric Oxide 102: 12-20. 
22. Pandit A (2005) The physiologic basis of high-altitude diseases. Annals of internal medicine 141(10):789-800.

23. Beall CM, Laskowski D, Erzurum SC (2012) nNitric oxide in adaptation to altitude. Free radical biology \& medicine 52(7): 1123-1134.

24. Abe K, Toba M, Alzoubi A, Ito M, Fagan KA, et al. (2010) Formation of plexiform lesions in experimental severe pulmonary arterial hypertension. Circulation 121(25): 2747-54.

25. Archer SL, Weir EK, Wilkins MR (2010) Basic science of pulmonary arterial hypertension for clinicians: new concepts and experimental therapies. Circulation 121(5): 2045-2066.

26. Cooney R, Hynes SO, Duffy AM, Sharif F, O’Brien T (2006) Adenoviralmediated gene transfer of nitric oxide synthase isoforms and vascular cell proliferation. Journal of vascular research 43(5): 462-72.

27. Radomski MW, Palmer RM, Moncada S (1990) An L-arginine/nitric oxide pathway present in human platelets regulates aggregation. Proceedings of the National Academy of Sciences of the United States of America 87(13): 5193-5197.

28. Cortese-Krott MM, Rodriguez-Mateos A, Sansone R, Kuhnle GG, ThasianSivarajah S, Krenz T, et al. (2012) Human red blood cells at work: identification and visualization of erythrocytic eNOS activity in health and disease. Blood 120(20): 4229-4237.

29. Kleinbongard P, Schulz R, Rassaf T, Lauer T, Dejam A et al. (2006) Red blood cells express a functional endothelial nitric oxide synthase. Blood 107(7): 2943-2951.

30. Förstermann U, Münzel T (2006) Endothelial nitric oxide synthase in vascular disease: from marvel to menace. Circulation 113(13): 17081714.

31. Chen J, Luo J, Yang X, Ouyang M, Zhu T, et al. (2020) Expression of ApoA5 and its function in the right ventricular failing and remodeling secondary to pulmonary hypertension. J Cell Physiol 236(2): 1013-1024.

32. Campo A, Mathai SC, Le Pavec J, Zaiman AL, Hummers LK, et al. (2011) Outcomes of hospitalisation for right heart failure in pulmonary arterial hypertension. The European respiratory journal 38(2): 359-367.

33. Galiè N, Humbert M, Vachiery JL, Gibbs S, Lang I, et al. (2016) 2015 ESC/ERS Guidelines for the diagnosis and treatment of pulmonary hypertension: The Joint Task Force for the Diagnosis and Treatment of Pulmonary Hypertension of the European Society of Cardiology (ESC) and the European Respiratory Society (ERS): Endorsed by: Association for European Paediatric and Congenital Cardiology (AEPC), International Society for Heart and Lung Transplantation (ISHLT). European heart journal 37(1): 67-119.

34. Fu S, Ping P, Wang F, Luo L (2018) Synthesis, secretion, function, metabolism and application of natriuretic peptides in heart failure. Journal of biological engineering 12: 2 .

35. Santaguida PL, Don-Wauchope AC, Oremus M, McKelvie R, Ali U (2014) BNP and NT-proBNP as prognostic markers in persons with acute decompensated heart failure: a systematic review. Heart failure reviews 19(4): 453-470.

36. Forte TM, Ryan RO (2015) Apolipoprotein A5: Extracellular and Intracellular Roles in Triglyceride Metabolism. Current drug targets 16(12): 1274-1280.

37. Schaap FG, Rensen PC, Voshol PJ, Vrins C, van der Vliet HN, et al. (2004) ApoAV reduces plasma triglycerides by inhibiting very low density lipoprotein-triglyceride (VLDL-TG) production and stimulating lipoprotein lipase-mediated VLDL-TG hydrolysis. The Journal of biological chemistry 279(27): 27941-27947.

38. Wang Y, Duo D, Yan Y, He R, Wang S, et al. (2020) Extract of Salvia przewalskii Repair Tissue Damage in Chronic Hypoxia Maybe through the RhoA-ROCK Signalling Pathway. Biological \& pharmaceutical bulletin 43(3): 432-439.
39. Wang Y, Duo D, Yan Y, He R, Wu X (2020) Magnesium lithospermate B ameliorates hypobaric hypoxia-induced pulmonary arterial hypertension by inhibiting endothelial-to-mesenchymal transition and its potential targets. Biomedicine \& Pharmacotherapy 130.

40. Nan X, Su S, Ma K, Ma X, Wang X, et al. (2018) Bioactive fraction of Rhodiola algida against chronic hypoxia-induced pulmonary arterial hypertension and its anti-proliferation mechanism in rats. Journal of ethnopharmacology 216: 175-83.

41. Jaitovich A, Jourd'heuil D (2017) A Brief Overview of Nitric Oxide and Reactive Oxygen Species Signaling in Hypoxia-Induced Pulmonary Hypertension. Advances in experimental medicine and biology 967: 7181.

42. Klinger JR, Abman SH, Gladwin MT (2013) Nitric oxide deficiency and endothelial dysfunction in pulmonary arterial hypertension. American journal of respiratory and critical care medicine 188(6): 639-646.

43. Beall CM (2007) Two routes to functional adaptation: Tibetan and Andean high-altitude natives. Proceedings of the National Academy of Sciences of the United States of America 104: 8655-8660.

44. Beall CM, Cavalleri GL, Deng L, Elston RC, Gao Y, et al. (2010) Natural selection on EPAS1 (HIF2alpha) associated with low hemoglobin concentration in Tibetan highlanders. Proceedings of the National Academy of Sciences of the United States of America 107(25): 1145911464.

45. Yang J, Jin ZB, Chen J, Huang XF, Li XM, et al. (2017) Genetic signatures of high-altitude adaptation in Tibetans. Proceedings of the National Academy of Sciences of the United States of America 114(16): 41894194.

46. Tamama K, Kawasaki H, Kerpedjieva SS, Guan J, Ganju RK, et al. (2011) Differential roles of hypoxia inducible factor subunits in multipotential stromal cells under hypoxic condition. Journal of cellular biochemistry 112(3): 804-817.

47. Patel SA, Simon MC (2008) Biology of hypoxia-inducible factor-2alpha in development and disease. Cell death and differentiation 15(4): 628-634.

48. Coulet F, Nadaud S, Agrapart M, Soubrier F (2003) Identification of hypoxia-response element in the human endothelial nitric-oxide synthase gene promoter. The Journal of biological chemistry 278(47): $46230-46240$

49. Ojiaku CA, Yoo EJ, Panettieri RA, (2017) Transforming Growth Factor $\beta 1$ Function in Airway Remodeling and Hyperresponsiveness. The Missing Link? American journal of respiratory cell and molecular biology 56(4): $432-442$

50. Liu Y, Cao Y, Sun S, Zhu J, Gao S, et al. (2016) Transforming growth factor-beta1 upregulation triggers pulmonary artery smooth muscle cell proliferation and apoptosis imbalance in rats with hypoxic pulmonary hypertension via the PTEN/AKT pathways. The international journal of biochemistry \& cell biology 77: 141-154.

51. Minchenko A, Bauer T, Salceda S, Caro J (1994) Hypoxic stimulation of vascular endothelial growth factor expression in vitro and in vivo. Laboratory investigation; a journal of technical methods and pathology 71(3): 374-379.

52. Goldberg MA, Schneider TJ (1994) Similarities between the oxygensensing mechanisms regulating the expression of vascular endothelial growth factor and erythropoietin. The Journal of biological chemistry 269(6): 4355-4359.

53. Caprara C, Grimm C (2012) From oxygen to erythropoietin: relevance of hypoxia for retinal development, health and disease. Progress in retinal and eye research 31(1): 89-119. 